$\operatorname{IPPP} / 12 / 87$

$\mathrm{DCPT} / 12 / 174$

January 20,2020

\title{
The LHC can probe small $x$ PDFs; the treatment of the infrared region
}

\author{
A.D. Martin ${ }^{a}$, E.G. de Oliveira ${ }^{a, b}$ and M.G. Ryskin ${ }^{a, c}$ \\ ${ }^{a}$ Institute for Particle Physics Phenomenology, University of Durham, Durham, DH1 3LE \\ ${ }^{b}$ Instituto de Física, Universidade de São Paulo, C.P. 66318,05315-970 São Paulo, Brazil \\ ${ }^{c}$ Petersburg Nuclear Physics Institute, NRC Kurchatov Institute, Gatchina, St. Petersburg, \\ 188300, Russia
}

\begin{abstract}
First, we show how to reduce the sensitivity of the NLO predictions of the DrellYan production of low-mass, lepton-pairs, at high rapidity, to the choice of factorization scale. In this way, observations of this process at the LHC can make direct measurements of parton distribution functions in the low $x$ domain; $x \lesssim 10^{-4}$. Second, we find an inconsistency in the conventional NLO treatment of the infrared region. We illustrate the problem using the NLO coefficient function of Drell-Yan production.
\end{abstract}

\section{LHC Drell-Yan production as a probe of low $x$}

The very high energy of the LHC allows a probe of the parton distribution functions (PDFs) of the proton at extremely small $x$, a region not accessible at previous accelerators. To extract the PDFs we describe the experimentally observed cross sections as a convolution of the PDFs and the cross section for the hard partonic subprocess, which is of the form

$$
d \sigma / d^{3} p=\int d x_{1} d x_{2} \operatorname{PDF}\left(x_{1}, \mu_{F}\right)\left|\mathcal{M}\left(p ; \mu_{F}, \mu_{R}\right)\right|^{2} \operatorname{PDF}\left(x_{2}, \mu_{F}\right),
$$

where a sum over the various pairs of PDFs is implied.

Here we focus on Drell-Yan production of a low mass $\mu^{+} \mu^{-}$pair. At LO the production of a $\mu^{+} \mu^{-}$system of mass $M$ and rapidity $Y$ arises from the subprocess $\gamma^{*} \rightarrow q \bar{q}$ with $x_{1,2}=$ 
$M \exp ( \pm Y) / \sqrt{s}$. So for $M=6 \mathrm{GeV}, Y=4$ (a domain which is accessible to the LHCb experiment) we probe $x_{1}=4.7 \times 10^{-2}, x_{2}=1.6 \times 10^{-5}$. The problem is that, in the low $x$ region, the PDFs strongly depend on the choice of the factorization scale $\mu_{F}$, see Fig. 1(a). It is made worse due to the dominance of the gluon PDF at small $x$, which means that the LO $q \bar{q} \rightarrow \gamma^{*}$ subprocess is overshadowed by the NLO subprocess $g q \rightarrow q \gamma^{*}$. However, it is this very dominance which will allow us to introduce a procedure which greatly suppresses the scale dependence of the predictions.

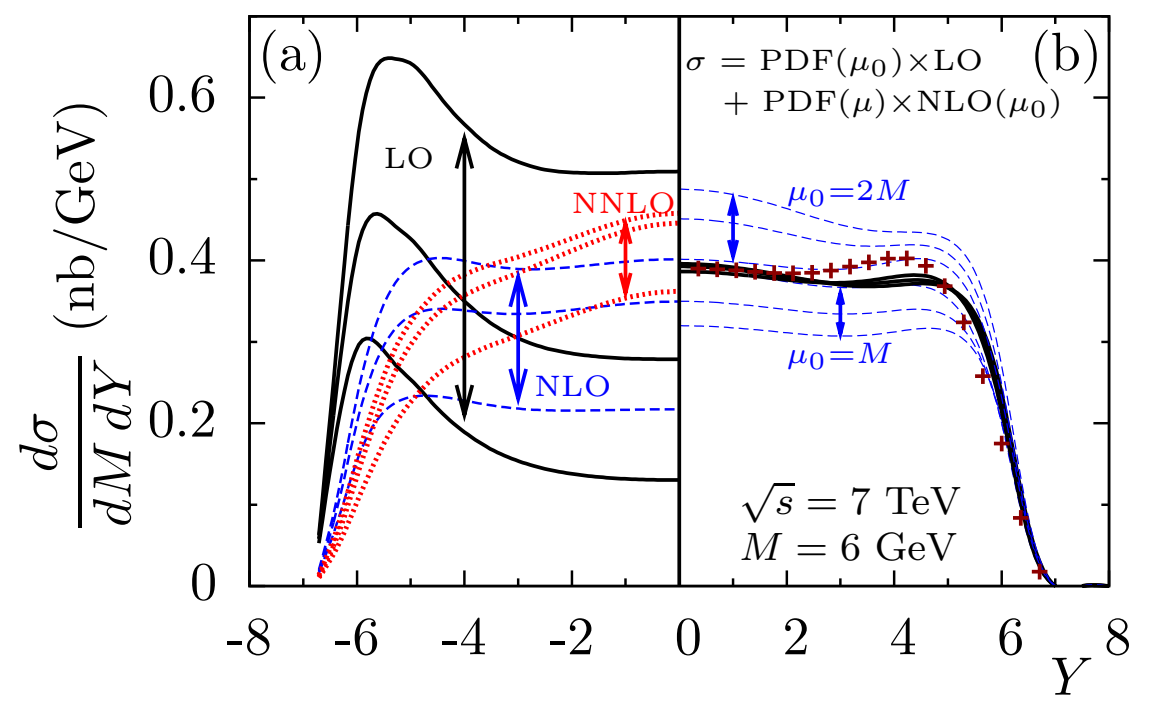

Figure 1: (a) Sensitivity of $M=6 \mathrm{GeV}$ Drell-Yan $\mu^{+} \mu^{-}$production, as a function of rapidity $Y$, to the choice of factorization scale: $\mu_{F}=M / 2, M, 2 M$, at LO, NLO, NNLO. (b) The bold lines correspond to the choice $\mu_{F}=\mu_{0}=1.4 M$ which minimizes $C_{\mathrm{rem}}^{\mathrm{NLO}}$, and show the stability with respect to the variations $\mu=M / 2, M, 2 M$ in the scale of the PDFs convoluted with $C_{\mathrm{rem}}^{\mathrm{NLO}}$ - the $\mu$ dependence is indicated by the symbolic equation at the top of the diagram. The dashed lines show that the stability disappears for other choices of $\mu_{0}$. The small crosses are the NNLO result. In this figure, taken from [1], the renormalization scale is fixed at $\mu_{R}=M$.

The plan is to choose the value of $\mu_{F}$ which minimizes the higher order $\alpha_{s}$ NLO, NNLO,.. contributions. To sketch the idea, we start with the LO expression for the cross section: $\sigma\left(\mu_{F}\right)=\operatorname{PDF}\left(\mu_{F}\right) \otimes C^{\mathrm{LO}} \otimes \operatorname{PDF}\left(\mu_{F}\right)$ in the collinear approach. The effect of varying the scale from $m$ to $\mu_{F}$, in both the left and right PDFs, can be expressed, to first order in $\alpha_{s}$, as

$$
\sigma\left(\mu_{F}\right)=\operatorname{PDF}(m) \otimes\left(C^{\mathrm{LO}}+\frac{\alpha_{s}}{2 \pi} \ln \left(\frac{\mu_{F}^{2}}{m^{2}}\right)\left(P_{\text {left }} C^{\mathrm{LO}}+C^{\mathrm{LO}} P_{\text {right }}\right)\right) \otimes \operatorname{PDF}(m),
$$

where the splitting functions $P_{\text {right }}=P_{q q}+P_{q g}$ and $P_{\text {left }}=P_{\bar{q} \bar{q}}+P_{\bar{q} g}$ act on the right and left PDFs respectively. We may equally well have incoming $\bar{q}$ 's in $P_{\text {right }}$ and incoming $q$ 's in $P_{\text {left }}$.

At NLO we may write

$$
\sigma\left(\mu_{F}\right)=\operatorname{PDF}\left(\mu_{F}\right) \otimes\left(C^{\mathrm{LO}}+\alpha_{s} C_{\mathrm{corr}}^{\mathrm{NLO}}\right) \otimes \operatorname{PDF}\left(\mu_{F}\right),
$$


where the $2 \rightarrow 2$ subprocesses $q \bar{q} \rightarrow g \gamma^{*}$ and $g q \rightarrow q \gamma^{*}$ are now calculated with better, than LLA, accuracy. However, we must subtract from $C^{\mathrm{NLO}}$, the part of the contribution already included, to LLA accuracy, in the $\alpha_{s}$ term in (2). The remaining contribution, $C_{\mathrm{rem}}^{\mathrm{NLO}}\left(\mu_{F}\right)$, now depends on the scale $\mu_{F}$, coming from the $\mu_{F}$ dependence of the LO LLA term that has been subtracted off. The trick is to choose an appropriate scale, $\mu_{F}=\mu_{0}$, so as to minimize the remaining NLO contribution $C_{\mathrm{rem}}^{\mathrm{NLO}}\left(\mu_{F}\right)$. Explicit calculation [1] shows that the optimum choice is $\mu_{F}=\mu_{0}=1.4 M$. The stability of the prediction, using MSTW NLO PDFs [2], is shown in Fig. 1(b). For $Y \gtrsim 3$, pure DGLAP PDF extrapolations become unreliable due to the absence of absorptive, $\ln (1 / x), .$. modifications. Rather, LHC data will provide a direct measure of PDFs in this low $x$ domain.

\section{Treatment of the infrared region in perturbative QCD}

Interestingly, a spin-off of the above study highlighted an inconsistency in the conventional treatment of the infrared region [3]. Again we use Drell-Yan as an example. For the main NLO subprocess we have

$$
\frac{d \hat{\sigma}\left(g q \rightarrow q \gamma^{*}\right)}{d|t|}=\frac{\alpha^{2} \alpha_{s} z}{9 M^{2}} \frac{1}{|t|}\left[\left((1-z)^{2}+z^{2}\right)+z^{2} \frac{t^{2}}{M^{4}}-2 z^{2} \frac{t}{M^{2}}\right]
$$

where $z=M^{2} / \hat{s}$ and $\sqrt{\hat{s}}$ is the incoming $g q$ c.m. energy. (Strictly speaking, $z$ is the ratio of the light-cone momentum fraction carried by the 'daughter' quark to that carried by the 'parent' gluon, $z=x_{q}^{+} / x_{g}^{+}$.) In order to calculate the inclusive cross section $d \sigma / d M^{2}$, it seems that we have to integrate over $t$ starting from $t=0$. If this were necessary, then we would face an infrared divergency.

However, we follow the procedure of the previous Section, which we call the physical approach. To avoid double-counting, we subtract the LO DGLAP-generated contribution. Then the remaining contribution of (4) is [3]

$$
\frac{d \hat{\sigma}_{\mathrm{rem}}^{\mathrm{NLO}}\left(g q \rightarrow q \gamma^{*}\right)}{d|t|}=\frac{\alpha^{2} \alpha_{s} z}{9 M^{2}} \frac{1}{|t|}\left[\left[(1-z)^{2}+z^{2}\right] \Theta\left(|t|-\mu_{F}^{2}\right)+z^{2} \frac{t^{2}}{M^{4}}-2 z^{2} \frac{t}{M^{2}}\right] .
$$

which has no singularity as $t \rightarrow 0$. The LO DGLAP evolution has accounted for all virtualities $|t|=k^{2}<\mu_{F}^{2}$; with the contribution of $k^{2}<Q_{0}^{2}$ hidden in the phenomenological input PDF at $Q^{2}=Q_{0}^{2}$.

On the other hand, the conventional prescription evaluates the inclusive cross section, $d \sigma / d M^{2}$, by integrating (4) over the infrared divergency at $t=0$ using $4+2 \epsilon$ dimensional space to regularize the integral [4, 5, 6]. Then the contribution from very small $t$ produces a $1 / \epsilon$ pole, which is absorbed into the incoming PDF. The conventional prescription is as follows. The same $g q \rightarrow q \gamma^{*}$ diagram, but now generated by LO DGLAP evolution, is considered; it gives an $1 / \epsilon$ pole which cancels the corresponding $1 / \epsilon$ pole in hard matrix element (coefficient 
function). However, we are left with $\epsilon / \epsilon$ terms of infrared origin, which produce a non-zero result as $\epsilon \rightarrow 0$. Unlike the finite $\epsilon / \epsilon$ terms of ultraviolet origin, which can be treated as pointlike counter-terms in the Lagrangian, the infrared $\epsilon / \epsilon$ contribution makes no physical sense in QCD theory, since the confinement eliminates any interaction at very large distances.

To be explicit, the conventional prescription gives

$$
\frac{M^{2} d \hat{\sigma}_{\mathrm{rem}}^{\mathrm{NLO}}\left(g q \rightarrow q \gamma^{*}\right)}{d M^{2}}=\frac{\alpha^{2} \alpha_{s} z}{9 M^{2}}\left\{\left[(1-z)^{2}+z^{2}\right] \ln \frac{(1-z)^{2}}{z}+\frac{1}{2}+3 z-\frac{7}{2} z^{2}\right\}
$$

which is to be compared with the result,

$$
\frac{M^{2} d \hat{\sigma}_{\mathrm{rem}}^{\mathrm{NLO}}\left(g q \rightarrow q \gamma^{*}\right)}{d M^{2}}=\frac{\alpha^{2} \alpha_{s} z}{9 M^{2}}\left\{\left[(1-z)^{2}+z^{2}\right] \ln \frac{(1-z)}{z}+\frac{1}{2}+z-\frac{3}{2} z^{2}\right\},
$$

obtained, from (5), using the physical approach. We have checked that the spurious $\epsilon / \epsilon$ contribution in the conventional approach is responsible for the difference,

$$
\frac{\alpha^{2} \alpha_{s} z}{9 M^{2}}\left\{\left[(1-z)^{2}+z^{2}\right] \ln (1-z)+2 z(1-z)\right\}
$$

between (6) and (7).

The message is that to get a reliable result we must use the physical approach and to subtract from (4) the $1 / t$ singularity exactly, so obtaining the non-singular expression (5). Dimensional regularisation is not appropriate in the infrared region. As an example we have used the NLO coefficient function for Drell-Yan production. However, the result applies more generally. It is relevant to other processes, such as deep inelastic scattering [3]. It is relevant to the NLO splitting functions.

It should be noted that the physical approach cannot be considered as an alternative factorization scheme. That is, the difference between the conventional and the physical coefficient functions cannot be compensated by a re-definition of the parton distributions (PDFs). The corresponding (infrared) $\epsilon / \epsilon$ corrections in the splitting functions do not coincide with those that come from the re-definition of the PDFs.

\section{Acknowledgements}

This work was supported by the grant RFBR 11-02-00120-a and by the Federal Program of the Russian State RSGSS-4801.2012.2; and by FAPESP (Brazil) under contract 2012/05469-4.

\section{References}

[1] E.G. de Oliveira, A.D. Martin and M.G. Ryskin, Eur. Phys. J C72, 2069 (2012). 
[2] A.D. Martin, W.J. Stirling, R.S. Thorne and G. Watt, Eur. Phys. J. C63, 189 (2009).

[3] E.G. de Oliveira, A.D. Martin and M.G. Ryskin, arXiv;1206.2223.

[4] G. Altarelli, R.K. Ellis and G. Martinelli, Nucl. Phys. B157, 461 (1979).

[5] See, for example, R.K. Ellis, W.J. Stirling and B.R. Webber, in QCD and Collider Physics (Cambridge Univ. Press, 1996) and refs. therein.

[6] C. Anastasiou, L.J. Dixon, K. Melnikov and F. Petriello, Phys. Rev. D69, 094008 (2004). 